\section{Sputtering technique forms versatile quasicrystalline coatings}

Dozens of quasicrystals with different compositions and symmetries have been discovered over the past 25 years. Most quasicrystalline structures result in ceramic-like properties such as high hardness, anti-corrosion, and non-stick properties. Due to their ceramic-like, brittle nature, research has focused practical applications on the development of quasicrystalline coatings. However, with the notable exception of the Al-Cu-Fe ternary system and the Al$\mathrm{Cu}-\mathrm{Fe}-\mathrm{Cr}$ and $\mathrm{Al}-\mathrm{Co}-\mathrm{Fe}-\mathrm{Cr}$ quaternary systems that are thermally stable up to $700^{\circ} \mathrm{C}$, many of the metallic combinations that have been found to exhibit quasicrystalline atomic structures and properties are thermally unstable, thus impractical for most applications.

The use of quasicrystalline coatings for non-stick cookware is a promising option for a large market that is undergoing fundamental change. As of 2009, the non-stick aluminum cookware market segment in the United States was estimated to be about $\$ 880$ million dollars, with a forecasted growth rate of $3.2 \%$ through 2014. The use of quasicrystalin cookware is made even more promising by a request from the Environmental

\section{The pitch} thermal and electrical resistance, high line coatings for non-stick applications
Protection Agency that cookware manufacturers eliminate perfluorooctanoic acid (PFOA) in non-stick coatings by 2015. Quasicrystalline coatings are PFOA-free.

The company Technology Assessment and Transfer (TA\&T) has conducted extensive research and development (R\&D) on these three quasicrystalline systems for a host of applications related to friction, wear and corrosion-resistant coatings. Compared with porous plasma spray coatings, the development, composition, and property optimization was based on magnetron sputtering. Magnetron sputtering is a versatile, highly controllable, vacuum coating process that enables quasicrystalline coatings to be applied to virtually any polymeric, metallic, and ceramic substrate or component. Magnetron sputtering offers precise control of coating density, structure, thickness, and properties. Measurements of film compositions, x-ray and transmission electron microscopy diffraction patterns, surface energies, friction coefficients, wear, and corrosion rates were correlated with a variety of sputtering conditions for both the Al-Cu-Fe ternary and the two $\mathrm{Al}$ rich quaternary phases in both the as-deposited state and post

\section{The technology}

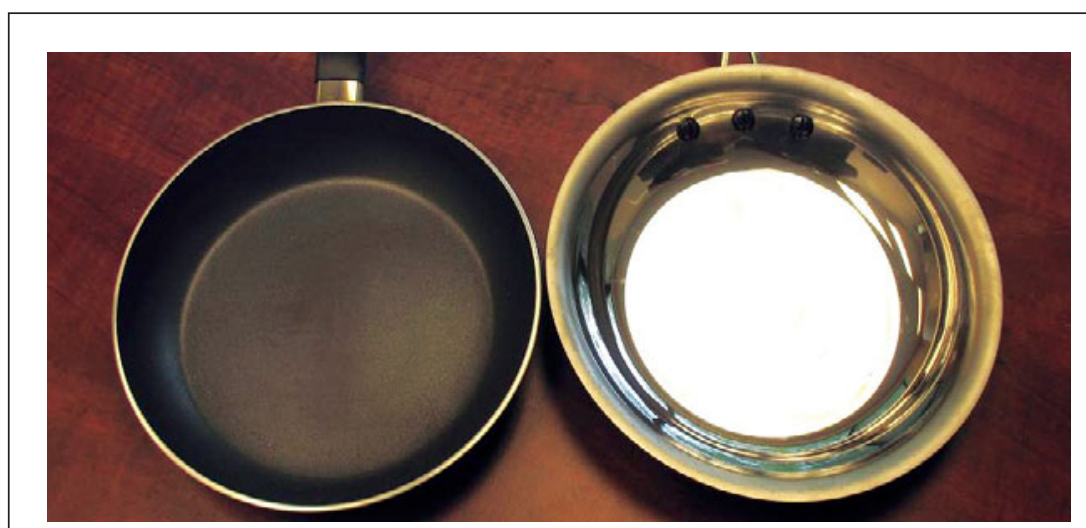

A quasicrystalline-coated pan (right) compared with a Teflon-coated pan (left). annealed conditions.

Adjustments were made in both target compositions and sputtering conditions to achieve dense, adherent, stable films and coatings of the desired icosahedral (5-fold) Al-Cu-Fe quasicrystalline structure and the 10-fold decagonal symmetries of the $\mathrm{Al}-\mathrm{Cu}-\mathrm{Fe}-\mathrm{Cr}$ and Al-Co-Fe-Cr quaternary compositions. High-temperature anneals for varying lengths of time in both vacuum and air demonstrate that bulk quasicrystalline properties are still retained despite the inclusion of some oxygen.

One of the major breakthroughs that allowed the production of high-quality quasicrystalline sputtered films was the development of crack-free, hot-pressed quasicrystalline sputtering targets. The extreme brittle nature of quasicrystalline materials caused severe cracking of the sputtering targets in the initial trials. (TA\&T has a patent on their sputtering target innovation.) The anti-stick, antiwear properties of TA\&T's sputtered quasicrystalline coatings have been demonstrated with frying pans. The figure shows a sputtered quasicrystalline-coated frying pan compared to a commercial Teflon-coated frying pan.

Other potential applications include selective solar absorbers for powerconversion, broad wavelength reflectors, and low-friction and corrosion-resistant biocompatibility for bone repair and prostheses applications. The magnetron sputtering capability can be readily applied to other stable quasicrystalline alloys such as AlPdMn.

\footnotetext{
Opportunities

TA\&T is interested in developing cooperative research and development efforts as well as offering potential licensing arrangements. Source: Larry Fehrenbacher, Technology Assessment and Transfer, Inc., 133 Defense Highway, Suite 212, Annapolis, MD 21410 USA; tel. 410-224-3710; e-mail larry@techassess.com; and www.techassess.com.
} 